\title{
Nonlinear Dynamics of FPU Lattice Type Protein Molecular Chain
}

\author{
K. Angelin Jeba ${ }^{1}$, M. M. Latha ${ }^{2}$ \\ ${ }^{1}$ (Department of Physics, Women's Christian College, Nagercoil, India) \\ ${ }^{2}$ (Department of Physics, Women's Christian College, Nagercoil, India)
}

\begin{abstract}
We explore the dynamical consequences of a protein molecular system by proposing a quartic $\beta$ Fermi-Pasta-Ulam lattice model. The nonlinear terms in the model Hamiltonian are quantized using suitable auxiliary relations resulting in a quantized number conserving FPU Hamiltonian. The resulting dynamical equation of motion is analysed in the continuum limit numerically which gives rise to a similariton solution. The amplitude of similariton increases significantly whereas the width of the similariton decreases with respect to lattice parameter.
\end{abstract}

Keywords: FPU lattice, Nonlinear Dynamics, Protein molecular chain, Similariton

\section{Introduction}

Many biological processes are associated with the bioenergy transport through protein molecules, where energy is released by the hydrolysis of Adenosine Triphosphate (ATP) molecules which amounts to about $0.4 \mathrm{eV}$. Within each peptide group, quantum transitions occur due to the vibrational structure of the $\mathrm{C}=\mathrm{O}$ double bond (amide-I) in the region of the infrared frequency spectrum. Phonons will propagate through the system from one group to another, thereby introducing dispersive effects. Davydov [1] considered a mathematical model to study the energy transfer in alpha-helical proteins, and he showed that the transport of the hydrolysis energy of ATP along alpha-helical proteins is through the formation of solitons moving without loss of energy [2-5]. In all the works [6-12] that followed Davydov, the dynamics is studied by proposing suitable Hamiltonian including interactions of different types such as molecular excitations, dipole-dipole interactions, vibrational excitations, coupling between exciton and phonon, quadrupole-quadrupole type interactions, interspine coupling etc. The dynamics associated with the above models is found to be governed by nonlinear Schrödinger type equations. Recently some works [13-15] have been reported on the study of phonon (vibron) propagation in protein molecules by considering a linear lattice.

The one dimensional nonlinear model introduced by Fermi, Pasta and Ulam (FPU) [16] in 1955 has proved to be a backbone of nonlinear dynamics during the last half century. This type of lattice has not been considered while studying the nonlinear dynamics of protein molecular systems. Hence in this work, we investigate the dynamics of a protein molecular system by considering a FPU type lattice. We propose the Model Hamiltonian and remodel it using the second quantized operators of quantum mechanics and construct the equations of motion. The solution to the resulting equations are constructed numerically and explained graphically.

The plan of the paper is as follows: In section 2, we describe the Hamiltonian and derive the equations of motion. The solution is plotted for different values of lattice parameters and the results are discussed in section 3 and concluded in section 4 .

\section{Hamiltonian of the $\beta$-Fermi Pasta Ulam Model}

We consider a one dimensional protein molecular chain described through the Hamiltonian

$$
H=\sum_{n} \frac{p_{n}^{2}}{2 M}+\frac{C}{2}\left(x_{n}-x_{n-1}\right)^{2}+\frac{b}{4}\left(x_{n}-x_{n-1}\right)^{4}
$$

where $x_{n}$ is the position and $p_{n}$ is the momentum of the n-th particle. $C, b$ denote the strength of the linear and nonlinear acoustic nearest-neighbour coupling respectively. This Hamiltonian corresponds to the classical $\beta$ - FPU model and has been investigated in numerous works. In order to quantise equation (1) we introduce phonon creation and annihilation operators $a_{n}^{\dagger}, a_{n}$ respectively. The position and momentum operators of the Hamiltonian in the discrete position are: 


$$
\begin{aligned}
& p_{n}=i \sqrt{\frac{\hbar M \omega}{2}}\left(a_{n}^{\dagger}-a_{n}\right) \\
& x_{n}=\sqrt{\frac{\hbar}{2 M \omega}}\left(a_{n}^{\dagger}+a_{n}\right)
\end{aligned}
$$

Next, we proceed with the inclusion of the nonlinear terms of the Hamiltonian. For this, we use the following auxillary relations [17]:

$$
\sum_{n}\left(x_{n}-x_{n-1}\right)^{2}=\sum_{n} x_{n}^{2}+\sum_{n} x_{n-1}^{2}-2 \sum x_{n} x_{n-1}
$$

which by virtue of lattice periodicity and by assuming $N \rightarrow \infty$, becomes:

Similarly

$$
\sum_{n}\left(x_{n}-x_{n-1}\right)^{2}=2 \sum_{n} x_{n}^{2}-2 \sum_{n} x_{n}\left(x_{n+1}+x_{n-1}\right)
$$

$$
\sum_{n}\left(x_{n}-x_{n-1}\right)^{4}=2 \sum_{n} x_{n}^{4}-4 \sum_{n} x_{n}^{3}\left(x_{n+1}+x_{n-1}\right)+3 \sum_{n} x_{n}^{2}\left(x_{n+1}^{2}+x_{n-1}^{2}\right)
$$

Using equations (5) and (6) in the Hamiltonian given in equation (1), the discrete Hamiltonian turns out to be:

$$
\begin{aligned}
& H=\sum_{n}\left[\frac{p_{n}^{2}}{2 M}+a\left(\frac{\hbar}{2 M \omega}\right)^{2}\left(a_{n}^{\dagger} a_{n}^{\dagger}+a_{n}^{\dagger} a_{n}+a_{n} a_{n}^{\dagger}+a_{n}^{2}+a_{n}^{\dagger} a_{n+1}^{\dagger}+a_{n}^{\dagger} a_{n+1}+\right.\right. \\
& \left.a_{n} a_{n+1}^{\dagger}+a_{n} a_{n+1}+a_{n}^{\dagger} a_{n-1}^{\dagger}+a_{n}^{\dagger} a_{n-1}+a_{n} a_{n-1}^{\dagger}+a_{n} a_{n-1}\right)+b\left(\frac{\hbar}{4 M \omega}\right)^{2} \\
& \left(a_{n-1}^{2} a_{n}^{2} a_{n-1} a_{n}^{3}+a_{n}^{4}+a_{n}^{3} a_{n+1}+a_{n}^{2} a_{n+1}^{2}+2 a_{n-1} a_{n}^{2} a_{n-1}^{\dagger}+a_{n}^{3} a_{n-1}^{\dagger}+\right. \\
& a_{n}^{2} a_{n-1}^{\dagger 2}+2 a_{n-1}^{2} a_{n} a_{n}^{\dagger}+3 a_{n-1} a_{n}^{2} a_{n}^{\dagger}+4 a_{n}^{3} a_{n}^{\dagger}+3 a_{n}^{2} a_{n+1} a_{n}^{\dagger}+2 a_{n} a_{n+1}^{2} a_{n}^{\dagger}+ \\
& 4 a_{n-1} a_{n} a_{n-1}^{\dagger} a_{n}^{\dagger}+3 a_{n}^{2} a_{n-1}^{\dagger} a_{n}^{\dagger}+2 a_{n} a_{n-1}^{\dagger 2} a_{n}^{\dagger}+a_{n-1}^{2} a_{n}^{\dagger 2}+3 a_{n-1} a_{n} a_{n}^{\dagger 2}+ \\
& 6 a_{n}^{2} a_{n}^{\dagger 2}+3 a_{n} a_{n+1} a_{n}^{\dagger 2}+a_{n+1}^{2} a_{n}^{\dagger 2}+2 a_{n-1} a_{n-1}^{\dagger} a_{n}^{\dagger 2}+3 a_{n} a_{n-1}^{\dagger} a_{n}^{\dagger 2}+a_{n-1}^{\dagger 2} a_{n}^{\dagger 2}+ \\
& a_{n-1} a_{n}^{\dagger 3}+4 a_{n} a_{n}^{\dagger 3}+a_{n+1} a_{n}^{\dagger 3}+a_{n-1}^{\dagger} a_{n}^{\dagger 3}+a_{n}^{\dagger 4}+a_{n}^{3} a_{n+1}^{\dagger}+2 a_{n}^{2} a_{n+1} a_{n+1}^{\dagger}+ \\
& 3 a_{n}^{2} a_{n}^{\dagger} a_{n+1}^{\dagger}+4 a_{n} a_{n+1} a_{n}^{\dagger} a_{n+1}^{\dagger}+3 a_{n} a_{n}^{\dagger 2} a_{n+1}^{\dagger}+2 a_{n+1} a_{n}^{\dagger 2} a_{n+1}^{\dagger}+a_{n}^{\dagger 3} a_{n+1}^{\dagger}+ \\
a_{n}^{2} a_{n+1}^{\dagger 2}+ & \left.\left.2 a_{n} a_{n}^{\dagger} a_{n+1}^{\dagger 2}+a_{n}^{\dagger 2} a_{n+1}^{\dagger 2}\right)\right]
\end{aligned}
$$

Having constructed the Hamiltonian for the different interactions, we derive the equations of motion for the dynamical variables $a_{n}$ which is given by:

$$
i \hbar \frac{d a_{n}}{d t}=\left[a_{n}, H\right]
$$

where $a_{n}$ satisfies the commutation relations $\left[a_{n}, a_{n}^{\dagger}\right]=1$. Let us for this moment introduce the normalization conditions $\sum_{n}\left|a_{n}\right|^{2}=1$. The equation of motion becomes:

$$
\begin{aligned}
& i \hbar \frac{d a_{n}}{d t}=c_{1}\left(a_{n}^{*}-a_{n}\right)+c_{2}\left(a_{n+1}^{*}+a_{n}^{*}+a_{n-1}^{*}+a_{n+1}+a_{n}+a_{n-1}\right)+c_{3}\left(4 a_{n} a_{n+1}^{2}+\right. \\
& 4 a_{n} a_{n-1}^{2}+a_{n+1}^{3}+3 a_{n}^{2} a_{n-1}+4 a_{n}^{3}+3 a_{n}^{2} a_{n+1}+a_{n-1}^{3}+4 a_{n} a_{n-1} a_{n-1}^{*}+2 a_{n+1}^{2} a_{n}^{*} \\
& +3 a_{n}^{2} a_{n-1}^{*}+4 a_{n} a_{n-1}^{* 2}+4 a_{n} a_{n+1} a_{n+1}^{*}+2 a_{n-1}^{2} a_{n}^{*}+6 a_{n} a_{n-1} a_{n}^{*}+6 a_{n} a_{n-1} a_{n}^{*} \\
& +3 a_{n+1}^{2} a_{n+1}^{*}+12 a_{n}^{2} a_{n}^{*}+6 a_{n} a_{n+1} a_{n}^{*}+3 a_{n-1}^{2} a_{n-1}^{*}+2 a_{n+1}^{2} a_{n}^{*}+4 a_{n-1} a_{n} a_{n}^{*} \\
& +4 a_{n-1} a_{n-1}^{*} a_{n}^{*}+4 a_{n+1} a_{n}^{*} a_{n+1}^{*}+6 a_{n} a_{n-1}^{*} a_{n}^{*}+4 a_{n-1}^{* 2} a_{n}^{*}+4 a_{n} a_{n+1}^{* 2}+ \\
& 3 a_{n-1} a_{n}^{* 2}+3 a_{n+1} a_{n+1}^{* 2}+12 a_{n} a_{n}^{* 2}+3 a_{n+1} a_{n}^{* 2}+3 a_{n-1} a_{n-1}^{* 2}+4 a_{n}^{*} a_{n+1}^{* 2}
\end{aligned}
$$




$$
\begin{aligned}
& +3 a_{n-1}^{*} a_{n}^{* 2}+a_{n+1}^{* 3}+4 a_{n}^{* 3}+a_{n-1}^{3}+3 a_{n}^{2} a_{n+1}^{*}+4 a_{n} a_{n+1} a_{n+1}^{*}+2 a_{n-1}^{2} a_{n}^{*} \\
& \left.+6 a_{n} a_{n}^{*} a_{n+1}^{*}+4 a_{n+1} a_{n}^{*} a_{n+1}^{*}+4 a_{n-1} a_{n-1}^{*} a_{n}^{*}+3 a_{n}^{*} a_{n+1}^{*}\right)
\end{aligned}
$$

where $c_{1}=\frac{p_{n}^{2}}{2 M}, c_{2}=a\left(\frac{\hbar}{2 M \omega}\right)^{2}$ and $c_{3}=b\left(\frac{\hbar}{4 M \omega}\right)^{2}$.

Equation (9) describes the dynamics of alpha-helical proteins in the discrete form. Due to nonlinearity and discreteness, it is difficult to solve equation (9) in its present form. Hence we transform equation (9) into a continuum equation using Taylor's series. The Taylor series expansion is of the form:

$$
a_{n \pm 1}=a \pm \gamma a_{x}+\frac{1}{2} \gamma^{2} a_{x x} \pm \frac{1}{6} \gamma^{3} a_{x x x}+\frac{1}{24} \gamma^{4} a_{x x x x}
$$

After using Taylor series expansion, equation (9) reduces to:

$$
\begin{aligned}
& i \hbar \frac{d a}{d t}=a c_{1}+3 a c_{2}+20 a^{3} c_{3}+c_{1} a^{*}+3 c_{2} a^{*}+60 a^{2} c_{3} a^{*}+60 a c_{3} a^{* 2}+ \\
& 20 c_{3} a^{* 3}+4 a^{2} c_{3} \gamma a_{x}^{*}+\gamma^{2}\left(14 a c_{3} a_{x}^{2}+c_{2} a_{x x}+14 a^{2} c_{3} a_{x x}+14 c_{3} a_{x}^{2} a^{*}+\right. \\
& 28 a c_{3} a_{x x} a^{*}+14 c_{3} a_{x x} a^{* 2}+24 a c_{3} a_{x} a_{x}^{*}+28 c_{3} a_{x} a^{*} a_{x}^{*}+14 a c_{3} a_{x}^{* 2}+ \\
& \left.14 c_{3} a^{*} a_{x}^{* 2}+c_{2} a_{x x}^{*}+12 a^{2} c_{3} a_{x x}^{*}+28 a c_{3} a^{*} a_{x x}^{*}+14 c_{3} a^{* 2} a_{x x}^{*}\right)+ \\
& \gamma^{3}\left(2 a c_{3} a_{x x} a_{x}^{*}+2 a c_{3} a_{x} a_{x x}^{*}+\frac{2}{3} a^{2} c_{3} a_{x x x}^{*}\right)+\gamma^{4}\left(3 c_{3} a_{x}^{2} a_{x x}+\frac{7}{2} a c_{3} a_{x x}^{2}+\right. \\
& \frac{14}{3} a c_{3} a_{x} a_{x x x}+\frac{c_{2} a_{x x x}}{12}+\frac{7}{6} a^{2} c_{3} a_{x x x}+\frac{7}{2} c_{3} a_{x x}^{2} a^{*}+\frac{14}{3} c_{3} a_{x} a_{x x x} a^{*}+ \\
& \frac{7}{3} a c_{3} a_{x x x} a^{*}+\frac{7}{6} c_{3} a_{x x x x} a^{* 2}+6 c_{3} a_{x} a_{x x} a_{x}^{*}+4 a c_{3} a_{x x x} a_{x}^{*}+\frac{14}{3} c_{3} a_{x x x} a^{*} a_{x}^{*} \\
& +3 c_{3} a_{x x} a_{x}^{* 2}+3 c_{3} a_{x}^{2} a_{x x}^{*}+6 a c_{3} a_{x x} a_{x x}^{*}+7 c_{3} a_{x x} a^{*} a_{x x}^{*}+6 c_{3} a_{x} a_{x}^{*} a_{x x}^{*} \\
& +3 c_{3} a_{x}^{* 2} a_{x x}^{*}+\frac{7}{2} a c_{3} a_{x x}^{* 2}+\frac{7}{2} c_{3} a^{*} a_{x x}^{* 2}+4 a c_{3} a_{x} a_{x x x}^{*}+\frac{14}{3} c_{3} a_{x} a^{*} a_{x x x}^{*}+ \\
& \frac{14}{3} a c_{3} a_{x}^{*} a_{x x x}^{*}+\frac{14}{3} c_{3} a^{*} a_{x}^{*} a_{x x x}^{*}+\frac{1}{12} c_{2} a_{x x x x}^{*}+a^{2} c_{3} a_{x x x x}^{*}+ \\
& \left.\frac{7}{3} a c_{3} a^{*} a_{x x x x}^{*}+\frac{7}{6} c_{3} a^{* 2} a_{x x x x}^{*}\right)
\end{aligned}
$$

In equation (11) the variable $a$ is a complex quantity. To write the equation in terms of real variables, we use the substitution $a=u+i v$. After separating the real and imaginary parts equation (11) becomes:

$$
\begin{aligned}
& v_{t}=-\frac{1}{\hbar}\left[c_{3} \gamma^{4} v u_{x x}^{2}+\frac{4}{3} c_{3} \gamma^{3} u v u_{x x x}-\frac{4}{3} c_{3} \gamma^{4} v u_{x} u_{x x x}-\frac{1}{3} c_{3} \gamma^{4} u v u_{x x x x}-\right. \\
& 4 c_{3} u^{2} v_{x}+4 c_{3} v^{2} v_{x}-4 c_{3} \gamma^{2} v v_{x}^{2}+2 c_{3} \gamma^{2} u v_{x x}-2 c_{3} \gamma^{2} v^{2} v_{x x}+ \\
& 4 c_{3} \gamma^{3} v v_{x} v_{x x}-c_{3} \gamma^{4} v v_{x x}^{2}-\frac{2}{3} c_{3} \gamma^{3} u^{2} v_{x x x}+\frac{2}{3} c_{3} \gamma^{3} v^{2} v_{x x x}- \\
& \left.\frac{4}{3} c_{3} \gamma^{4} v v_{x} v_{x x x}+\frac{1}{6} c_{3} \gamma^{4} u^{2} v_{x x x}-\frac{1}{6} c_{3} \gamma^{4} v^{2} v_{x x x x}\right] \\
& u_{t}=\frac{1}{\hbar}\left[6 c_{2} u+160 c_{3} u^{3}+4 c_{3} u^{2} u_{x}-4 c_{3} v^{2} u_{x}+108 c_{3} \gamma^{2} u u_{x}^{2}+2 c_{2} \gamma^{2} u_{x x}+\right. \\
& 110 c_{3} \gamma^{2} u^{2} u_{x x}+2 c_{3} \gamma^{2} v^{2} u_{x x}+4 c_{3} \gamma^{3} u u_{x} u_{x x}+24 c_{3} \gamma^{4} u_{x}^{2} u_{x x}+ \\
& 27 c_{3} \gamma^{4} u u_{x x}^{2}+\frac{2}{3} c_{3} \gamma^{3} u^{2} u_{x x x}-\frac{2}{3} c_{3} \gamma^{3} v^{2} u_{x x x}+36 c_{3} \gamma^{4} u u_{x} u_{x x x}+
\end{aligned}
$$




$$
\begin{aligned}
& \frac{1}{6} c_{2} \gamma^{4} u_{x x x x}+\frac{55}{6} c_{3} \gamma^{4} u^{2} u_{x x x x}+\frac{1}{6} c_{3} \gamma^{4} v^{2} u_{x x x x}+8 c_{3} \gamma u v v_{x}- \\
& 4 c_{3} \gamma^{3} u v_{x} v_{x x}-c_{3} \gamma^{4} u v_{x x}^{2}+\frac{4}{3} c_{3} \gamma^{3} u v v_{x x x}-\frac{4}{3} c_{3} \gamma^{4} u v_{x} v_{x x x}- \\
& \frac{1}{3} c_{3} \gamma^{4} u v v_{x x x x}-2 c_{1} v+8 c_{3} \gamma u v u_{x}-4 c_{3} \gamma^{2} u_{x}^{2}-4 c_{3} \gamma^{2} u v u_{x x} \\
& \left.+4 c_{3} \gamma^{3} v u_{x} u_{x x}\right]
\end{aligned}
$$

Equations (12) and (13) represent the dynamics of alpha-helical proteins in the continuum limit.

\section{Results And Discussions}

The set of coupled equations (12) and (13) is solved numerically using MATHEMATICA. Figs. (1)-(4) represent the solutions for different values of the lattice parameter $\gamma$. The other parameter values are chosen to be $\mathrm{M}=1.91 \times 10^{-27} \mathrm{~kg}$ and $\mathrm{k}=13 \mathrm{~N} / \mathrm{m}$. It is seen from the figures that the phonon propagation through alpha-helical proteins is in the form of similaritons. Fig. (1) is for $\gamma=0.1$. The amplitude of similariton is found to increase with time but localized in space.

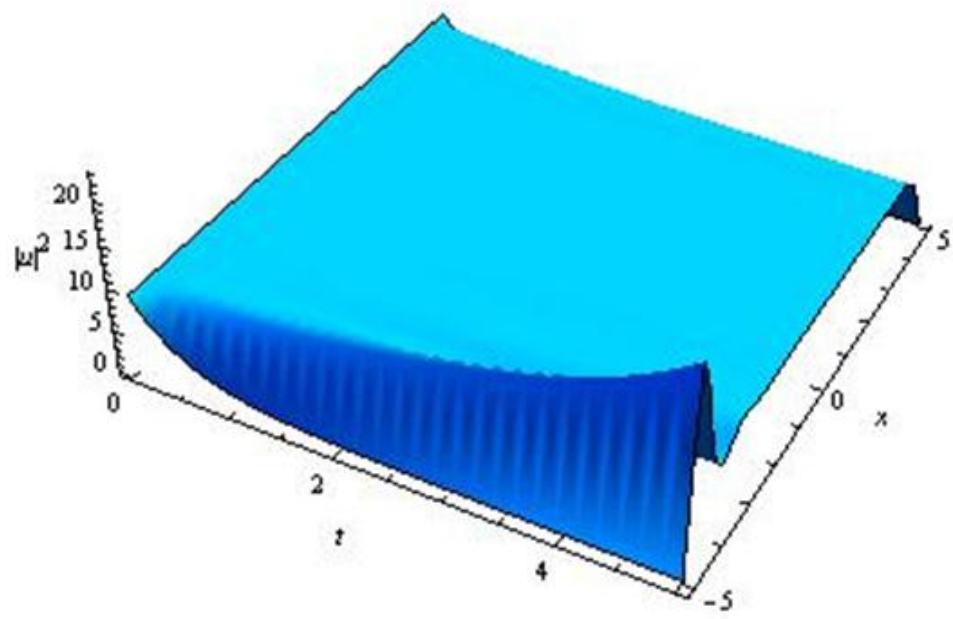

Figure 1: Similariton profile for $\gamma=0.1$

Figure (2) portrays the dynamics of alpha-helical proteins for $\gamma=0.5$. It shows that the amplitude of the profile is increased drastically for a small change in lattice parameter. The width of the similariton is decreased and pulse formation is enhanced.

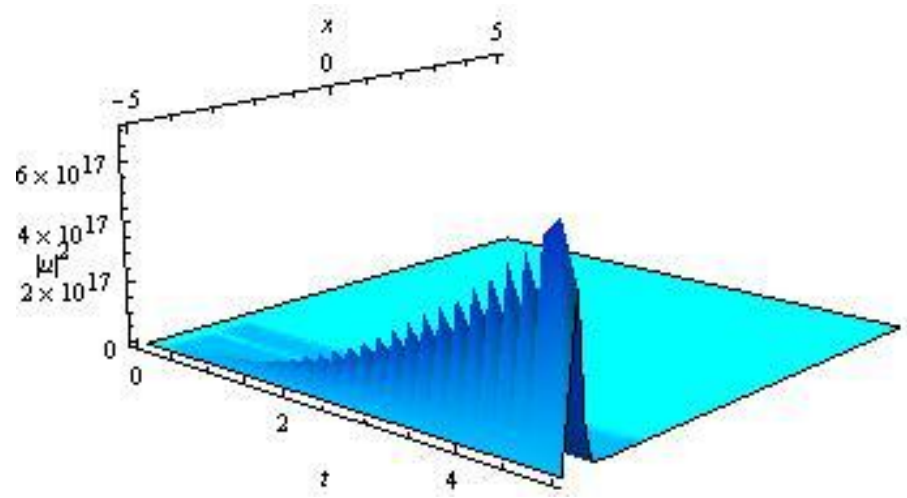

Figure 2: Similariton profile for $\gamma=0.5$

Figures (3) and (4) correspond to $\gamma=0.8$ and $\gamma=1$ respectively. From the figures it can be visualized that as the lattice parameter increases phonon propagation in alpha-helical protein system is highly affected which is noticed from the sudden decrease in amplitude. 


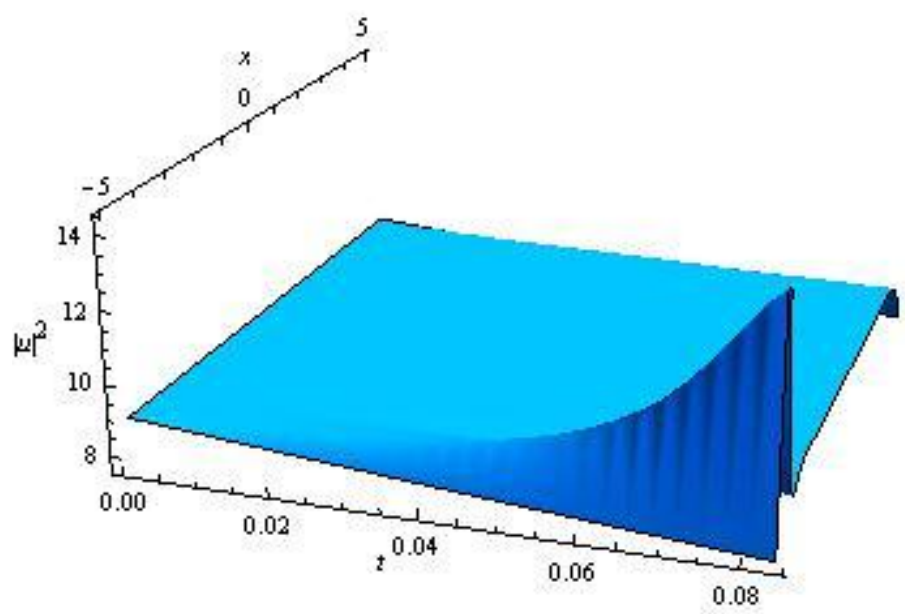

Figure 3: Similariton profile for $\gamma=0.8$

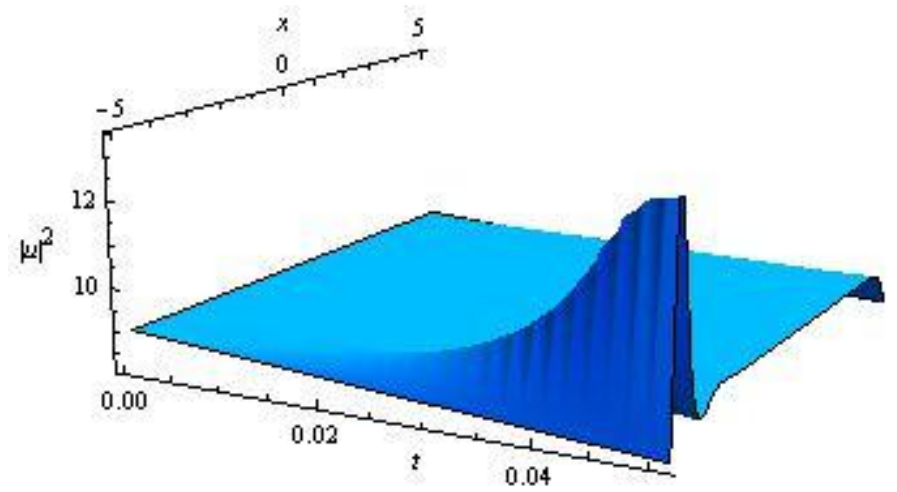

Figure 4: Similariton profile for $\gamma=1$.

\section{Conclusion}

In this paper we study the nature of phonon propagation in a FPU type alpha-helical protein lattice by constructing a suitable Hamiltonian and remodeling it using the second quantized operators of quantum mechanics. The dynamics is studied for different values of lattice parameters. The results show that the propagation is in the form of similariton. It is seen from the results that the dynamics highly depends on the lattice parameter.

\section{References}

[1]. A. S. Davydov and N.I. Kisluka, Phys. Stat. Sol. b 59, 1973, 465.

[2]. A.S. Davydov, Biofizika 19, 1974, 670.

[3]. A.S. Davydov, Studia Biophyzika 47, 1974, 221.

[4]. A. S. Davydov, A. A. Eremko and A. I. Segienko, Ukr. Fiz. Zh 23, 1978, 983.

[5]. A. S. Davydov, Solitons in molecular systems, (Reidel, Dordrecht, 1985).

[6]. W. F"orner, J. Mol. Model. 3, 1997, 78.

[7]. M. Daniel and M. M. Latha, Phys. Lett.A 252, 1999, 92.

[8]. M. Daniel and M. M. Latha, Phys. Lett. A 302, 2002, 94.

[9]. J. Edler, R. Pfister, V. Pouthier, C. Falvo and P. Hamm, Phys. Rev. Lett. 93, 2004, 106405.

[10]. K. C. Biswas, J. Gillespie, F. Majid, M. E. Edwards and A. Biswas, Advanced Studies in Biology 1, $2009,1$.

[11]. M. M. Latha and S. S. Veni, Phys. Scr 83, 2011, 035001.

[12]. S. S. Veni and M. M. Latha, Physica A 413, 2014, 93.

[13]. V. Pouthier, J. Chem. Phys. 118, 2003, 9364.

[14]. V. Pouthier, J. C. Light and C. Giardet, J. Chem. Phys. 114, 2004, 4955.

[15]. V. Pouthier and C. Falvo, Phys. Rev. E 69, 2004, 041906.

[16]. E. Fermi, J. R. Pasta and S. M. Ulam, Studies of nonlinear problems Vol. I (Los Alamos Report LA-1940, 1995)

[17]. Zoran Ivic and G. P. Tsironis, Physica D 216, 2006, 200. 